\title{
Anti-PD-1 Monoclonal Antibody LZM009
}

National Cancer Institute

\section{Source}

National Cancer Institute. Anti-PD-1 Monoclonal Antibody LZM009. NCI Thesaurus. Code C153149.

A recombinant, humanized monoclonal antibody directed against the negative immunoregulatory human cell surface receptor, programmed cell death 1 (PD-1), with potential immune checkpoint inhibitory and antineoplastic activities. Upon intravenous administration, LZM009 binds to PD-1 and inhibits the binding of PD-1 to the PD-1 ligands, programmed cell death-1 lig and 1 (PD-L1) and PD-1 ligand 2 (PD-L2). This prevents the activation of PD-1 and its downstream signaling pathways, leading to the activation of both $\mathrm{T}$-cells and $\mathrm{T}$-cell-mediated immune responses against tumor cells. PD-1 is a transmembrane protein in the immunoglobulin (Ig) superfamily expressed on activated $T$-cells that negatively regulates $T$-cell activation and effector function when activated by its ligands. PD-1 plays an important role in tumor evasion from host immunity. 